\title{
PKC $\varepsilon$ Phosphorylates and Mediates the Cell Membrane Localization of RhoA
}

\author{
Tizhi Su, ${ }^{1,2}$ Samuel Straight, ${ }^{3}$ Liwei Bao, ${ }^{4}$ Xiujie Xie, ${ }^{1,2}$ Caryn L. Lehner, ${ }^{5}$ Greg S. Cavey, ${ }^{5,6}$ \\ Theodoros N. Teknos, ${ }^{1,2}$ and Quintin Pan $^{1,2}$ \\ ${ }^{1}$ Arthur G. James Cancer Hospital and Richard J. Solove Research Institute, The Ohio State University Comprehensive Cancer Center, \\ Columbus, OH 43210, USA \\ ${ }^{2}$ Department of Otolaryngology-Head and Neck Surgery, The Ohio State University Wexner Medical Center, \\ Columbus, OH 43210, USA \\ ${ }^{3}$ Center for Live-Cell Imaging, Department of Microbiology and Immunology, University of Michigan, Ann Arbor, MI 48109, USA \\ ${ }^{4}$ Division of Hematology and Oncology, Department of Internal Medicine, University of Michigan Medical School, \\ Ann Arbor, MI 48108, USA \\ ${ }^{5}$ Van Andel Research Institute, Grand Rapids, MI 49503, USA \\ ${ }^{6}$ Southwest Michigan Innovation Center, Kalamazoo, MI 49008, USA
}

Correspondence should be addressed to Quintin Pan; Quintin.Pan@osumc.edu

Received 9 July 2013; Accepted 18 August 2013

Academic Editors: Y.-y. Liu and Y. Yu

Copyright (C) 2013 Tizhi Su et al. This is an open access article distributed under the Creative Commons Attribution License, which permits unrestricted use, distribution, and reproduction in any medium, provided the original work is properly cited.

\begin{abstract}
Protein kinase $\mathrm{C} \varepsilon(\mathrm{PKC} \varepsilon)$ signals through RhoA to modulate cell invasion and motility. In this study, the multifaceted interaction between $\mathrm{PKC} \varepsilon$ and RhoA was defined. Phosphopeptide mapping revealed that PKC $\varepsilon$ phosphorylates RhoA at T127 and S188. Recombinant PKCe bound to recombinant RhoA in the absence of ATP indicating that the association between PKC $\varepsilon$ and RhoA does not require an active ATP-docked $\mathrm{PKC} \varepsilon$ conformation. Activation of $\mathrm{PKC} \varepsilon$ resulted in a dramatic coordinated translocation of PKC $\varepsilon$ and RhoA from the cytoplasm to the cell membrane using time-lapse fluorescence microscopy. Stoichiometric FRET analysis revealed that the molecular interaction between PKCE and RhoA is a biphasic event, an initial peak at the cytoplasm and a gradual prolonged increase at the cell membrane for the entire time-course (12.5 minutes). These results suggest that the PKC $\varepsilon$ RhoA complex is assembled in the cytoplasm and subsequently recruited to the cell membrane. Kinase inactive (K437R) PKC $\varepsilon$ is able to recruit RhoA to the cell membrane indicating that the association between PKCe and RhoA is proximal to the active catalytic site and perhaps independent of a PKCe-RhoA phosphorylation event. This work demonstrates, for the first time, that $\mathrm{PKC} \varepsilon$ phosphorylates and modulates the cell membrane translocation of RhoA.
\end{abstract}

\section{Introduction}

Numerous publications have clearly defined the role of $\mathrm{PKC} \varepsilon$ as transforming oncogene in fibroblasts and epithelial cells. overexpression of $\mathrm{PKC} \varepsilon$ in $\mathrm{NIH} 3 \mathrm{~T} 3$ fibroblasts and FRC/TEX CL D rat colonic epithelial cells was shown to increase cell proliferation, enhance anchorage-independent colony formation, and induce a highly tumorigenic in vivo phenotype with tumor incidence of $100 \%[1,2]$. In addition, NIH3T3 fibroblasts with $\mathrm{PKC} \varepsilon$ overexpression were invasive and displayed a polarized morphology with extended long cellular membrane protrusions [3]. Epidermisspecific $\mathrm{PKC} \varepsilon$ transgenic mice developed highly malignant and metastatic squamous cell carcinomas in response to 12-O-tetradecanoylphorbol-13-acetate stimulation [4]. PKC $\varepsilon$ was demonstrated to transform androgen-dependent LNCaP prostate cancer cells into an androgen-independent variant [5]. Moreover, transgenic mice with selective overexpression of PKC $\varepsilon$ in the prostate epithelium developed prostate hyperplasia and prostate intraepithelial neoplasia [6]. Our laboratory demonstrated that inhibition of PKC $\varepsilon$ in MDA-MB231 cells, a highly metastatic breast cancer cell line with elevated $\mathrm{PKC} \varepsilon$ levels, was sufficient to dramatically decrease in vivo tumor growth and reduce the incidence of lung metastasis [7]. Subsequently, PKCe was shown to promote an invasive and 
motile phenotype in HNSCC through modulation of RhoA presumably through posttranslation phosphorylation [8].

RhoA, a member of the Rho GTPase family, has been implicated to be involved in the development and/or progression of numerous cancers. A recent report showed that overexpression of RhoA is sufficient to immortalize human mammary epithelial cells [9]. Elevated RhoA is associated with invasive breast cancer progression [10]. Moreover, miR31 was reported to be inversely associated with metastasis through inhibition of RhoA in breast cancer patients [11]. Multivariate analysis revealed that elevated RhoA is an independent prognostic biomarker of poorer overall survival in pancreatic adenocarcinoma [12]. High levels of RhoA correlated with venous invasion, advanced pTNM stage, and prognosis in hepatocellular carcinoma $[13,14]$. Increased RhoA is associated with tumor progression in ovarian carcinoma and lymph node metastasis and overall survival in bladder carcinoma $[15,16]$. Similarly, RhoA was shown to be biomarker for lymph node metastasis and overall survival in esophageal squamous cell carcinoma [17]. RhoA, Rac2, and Cdc42 were found to be elevated in premalignant dysplastic and HNSCC cell lines in comparison to normal keratinocytes [18]. Furthermore, based on their immunohistochemistry analyses, RhoA was suggested to be a promising biomarker of malignancy and/or aggressiveness in head and neck squamous cell carcinoma (HNSCC) [18].

Our previous work provided the initial evidence linking two proteins, $\mathrm{PKC} \varepsilon$ and RhoA, intimately involved in metastasis. PKCE was shown to signal through RhoA to modulate cell invasion and motility in HNSCC [8]. In this study, we further studied the interaction between PKCe and RhoA. $\mathrm{PKC} \varepsilon$ was shown to phosphorylate RhoA at T127 and S188. Interestingly, an active ATP-docked $\mathrm{PKC} \varepsilon$ conformation is not required for $\mathrm{PKC} \varepsilon$ to bind to RhoA indicating that the PKCE-RhoA complex is assembled independently of the transient substrate-kinase interaction at the catalytic site of PKCe. Stoichiometric FRET analysis with HEK293 cells overexpressing mCherry-PKC $\varepsilon$ and eGFP-RhoA revealed that the PKCe-RhoA complex is assembled in the cytoplasm and subsequently translocates to the cell membrane. Our work revealed that $\mathrm{PKC} \varepsilon$ phosphorylates RhoA but, intriguingly, also has a kinase-independent action to function as a chaperone to traffic RhoA to the cell membrane.

\section{Materials and Methods}

2.1. Plasmid Constructs. Human $\mathrm{PKC} \varepsilon \mathrm{cDNA}$ was cloned into pENTR/D-TOPO vector (Invitrogen, Carlsbad, CA) by PCR from a human cDNA library (Clontech, Mountain View, CA). The N-mCherry-tagged PKC $\varepsilon$ was made by inserting PKC $\varepsilon$ open reading frame into $\mathrm{BglII} / \mathrm{XbaI}$ site of mCherry-C1 vector (Clontech, Mountain View, CA). mCherry-PKCe/K437R mutant was generated using the QuikChange Lightning kit (Agilent Technologies, Inc., Santa Clara, CA). The positive control plasmid mCherry-linker-eGFP for stoichiometric FRET analysis was made by inserting mCherry DNA fragment into NheI/BglII sites and followed by eliminating the sequence between BamHl and BglII sites within the multiple cloning site in vector eGFP-C1 (Clontech), resulting in a 10 amino acid long in-frame linker SGLKDPPVAT.

2.2. Cell Line. HEK293 cells were purchased from ATCC (Rockville, MD) and cultured in Dulbecco's modified Eagle's medium supplemented with penicillin (100 units $/ \mathrm{mL}$ ), streptomycin $(100 \mu \mathrm{g} / \mathrm{mL})$, and $10 \%$ fetal bovine serum.

2.3. In Vitro Kinase Assay. Recombinant PKCe was incubated with recombinant RhoA in kinase buffer $(24 \mathrm{mM}$ Tris $(\mathrm{pH}$ 7.4), $0.5 \mathrm{mM}$ EDTA, $0.5 \mathrm{mM}$ EGTA, $10 \mathrm{mM} \beta$-mercaptoethanol, $1 \mu \mathrm{g} / \mathrm{mL}$ leupeptin, $1 \mu \mathrm{g} / \mathrm{mL}$ aprotinin, and $50 \mu \mathrm{g} / \mathrm{mL}$ PMSF) containing PKC activators, phosphatidylserine and diacylglycerol, and $\left[{ }^{32} \mathrm{P}\right]$ ATP for 30 minutes at $25^{\circ} \mathrm{C}$. Subsequently, termination buffer consisting of $7.5 \mathrm{M}$ guanidine$\mathrm{HCl}$ was added to stop the reaction. The incubation reaction was separated by SDS-PAGE and visualized using autoradiography.

2.4. Phosphopeptide Mapping. RhoA was phosphorylated by $\mathrm{PKC} \varepsilon$ in vitro and then subjected to digestion by trypsin, chymotrypsin, or Glu-C. Following enzyme digestion the sample was acidified to $0.5 \%$ trifluoroacetic acid concentration and stored at $-20^{\circ} \mathrm{C}$ until further analyzed. The digested RhoA protein was analyzed by reverse-phase nanoscale LC$\mathrm{MS}^{\mathrm{E}}$ using a Waters QTof Premier mass spectrometry system. Prior to analysis EDTA and diammonium phosphate were added to sample for a final concentration of $25 \mathrm{mM}$ each, and 11-25 ng of digested protein was analyzed. Peptides were separated using acetonitrile/water mobile phases containing $0.1 \%$ formic acid on a Waters NanoAcquity UPLC system employing a $300 \mu \mathrm{m}$ ID $\times 20 \mathrm{~mm}$ C-18 $5 \mu \mathrm{m}$ particle Symmetry trap column and a $75 \mu \mathrm{m} \mathrm{ID} \times 150 \mathrm{~mm}$ C-18 $1.7 \mu \mathrm{m} \mathrm{BEH}$ analytical column. Peptides were trapped for 15 minutes at $3 \mu \mathrm{L} / \mathrm{min}$ followed by gradient elution using $0-28 \%$ acetonitrile in 40 minutes through the analytical column at $300 \mathrm{~nL} / \mathrm{min}$. ESI was conducted at approximately $3.3 \mathrm{kV}$ using in-house prepared spray emitters. Emitters were constructed by sleeving a $7 \mathrm{~cm}$ piece of $20 \mu \mathrm{m}$ ID $\times 90$ OD FSC into a $3 \mathrm{~cm}$ piece of $100 \mu \mathrm{m}$ ID $\times 360$ OD FSC and gluing the junction with epoxy. The polyimide coating on the terminal end of the emitter was burned off using a microtorch, and the emitter was used with a Waters NanoEase ESI mount. The Qtof Premier mass spectrometer was programmed to collect alternate scan $M S^{\mathrm{E}}$ data as previously described $[19,20]$. Briefly, $\mathrm{MS}^{\mathrm{E}}$ data collection was performed by a low collision energy acquisition of 0.8 seconds followed by a high collision energy acquisition for 0.8 seconds without quadrupole mass filtering across a $50-1990 \mathrm{~m} / \mathrm{z}$ mass range. This was performed in an alternating fashion during a 65-minute run and termed LC$\mathrm{MS}^{\mathrm{E}}$ analysis. Low collision energy acquisition records all peptide precursor mass data, while the high collision energy portion of the acquisition collected peptide fragmentation data. Following the low collision energy acquisition set at 10 volts, collision energy was ramped from 10 volts to 40 volts over the 0.8 second high collision energy acquisition to accommodate peptides requiring different collision energy for fragmentation. Glu-fibrinopeptide at a concentration 
of $200 \mathrm{fmol} / \mu \mathrm{L}$ in $25 \%$ acetonitrile/water/ $0.1 \%$ formic acid was introduced as a lockspray calibrant through a second ESI probe at $0.5 \mu \mathrm{L} / \mathrm{min}$ using an auxiliary UPLC pump. Lockspray data was collected for 1 second every 30 seconds over a 65-minute analysis.

Following an LC-MS ${ }^{\mathrm{E}}$ analysis, data processing was performed using Waters PLGS software version 2.3 build 23 using the following parameters: low-energy threshold 100 counts, high-energy threshold 10 counts, and an intensity threshold of 1000 counts. Data processing combined the signal intensity of all charge states generated from a given peptides into singly charged $\mathrm{MH}^{+}$values and determined the peak apex for both low-energy precursors and all fragment ions within the vicinity of the precursor. This lockmass corrected, accurate mass data was used in two ways. First, the data was used to search Human RefSeq database version 17 within PLGS software using the following search parameters: peptide and fragment tolerance was automatic, the minimum fragment Ion matches per peptide were 3 , the minimum fragment ions per protein were 7 , the minimum peptides matches per protein were 1 , missed cleavages were 2 , the false positive rate was $4 \%$, and modifications allowed were Acetyl N-term, Carbamidomethyl-C, Carbamyl N-term, and phosphorylation at STY. Second, low-energy precursor $\mathrm{MH}^{+}$ data was copied into Excel and compared to $\mathrm{MH}^{+}$values calculated for predicted RhoA trypsin, chymotrypsin, or Glu$C$ protease peptides bearing up to 4 phosphate groups. (Lighthouse Data, GPMAW version 8.00srl, Odense, Denmark). Experimental $\mathrm{MH}^{+}$masses that matched within $0.03 \mathrm{Da}$ of GPMAW calculated values were evaluated manually in PLGS or Masslynx Protein/Peptide Editor software. Possible phosphopeptide assignment was made when the measured mass was within $0.03 \mathrm{Da}$ of the calculated phosphopeptide mass and greater than 3 accurate mass product ions could be assigned to a peptide sequence. Confident site-specific phosphorylation also used this criteria but further required fragment ions including phosphorylated serine or threonine amino acids.

2.5. Immunoprecipitation. Recombinant PKC $\varepsilon$ (GenWay Inc., San Diego, CA) was incubated with recombinant RhoA (Cytoskeleton, Denver, CO) in PKC kinase buffer for 30 minutes at $25^{\circ} \mathrm{C}$. The binding reaction was immunoprecipitated using agarose-conjugated anti-PKC $\varepsilon$ antibody or IgG (Abcam, Cambridge, MA) at $4^{\circ} \mathrm{C}$ with gentle agitation overnight. The suspension was centrifuged at $1,000 \times \mathrm{g}$ for 1 minute, and the agarose beads were washed three times with ice-cold PBS and resuspended in SDS sample buffer. The same procedure was performed to immunoprecipitate the binding reaction containing His-tagged PKCe-kinase domain (BioBasic Inc., Markham, Canada), and RhoA except agarose-conjugated anti-His antibody (Abcam) was used. The immunoprecipitated samples were boiled in SDS sample buffer, resolved by SDS-PAGE, and transferred to Immobilon membrane. The membranes were incubated with antiRhoA (Cytoskeleton) or anti-His (Millipore, Billerica, MA) antibodies and visualized by ECL using the Fast Western kit (Pierce, Rockford, IL).
2.6. Fluorescence Microscopy and Quantitative Stoichiometric FRET Analysis. HEK293 cells were seeded on $35 \mathrm{~mm}$ glassbottomed dishes one day prior to transfection with mCherry$\mathrm{PKC} \varepsilon$ and eGFP-RhoA. Fluorescence microscopy experiments were performed in the Center for Live-Cell Imaging (CLCI) at the University of Michigan Medical School using an Olympus IX70 inverted microscope (Olympus, Center Valley, $\mathrm{PA})$. Experiments involving live-cell imaging employed a heated stage (Harvard Apparatus, Inc., Holliston, MA) in combination with HEPES-buffered medium to maintain cell viability and activity for several hours of microscopic observation. Illumination was provided from a $100 \mathrm{~W}$ halogen lamp for phase-contrast microscopy and by an X-Cite 120 metal halide light source (EXFO, Mississauga, Canada) for fluorescent microscopy. The microscope was equipped with 100x (oil immersion; UPlan Fl, NA = 1.30), 40x (LCPLanFl, $\mathrm{NA}=0.6)$, and $10 \mathrm{x}(\mathrm{CPlan}, \mathrm{NA}=0.25)$ objectives. Excitation and emission filter sets (Chroma Technology Corp., Rockingham, VT) were used for fluorescent imaging; in particular set number $86014 \mathrm{v} 2$ includes filters used for GFP (excitation $492 \mathrm{~nm} / \mathrm{BP} 18$, emission $535 \mathrm{~nm} / \mathrm{BP} 40$ ) and mCherry (excitation $572 \mathrm{~nm} / \mathrm{BP} 23$, emission $630 \mathrm{~nm} / \mathrm{BP} 60$ ). The excitation and emission filters were mounted in a Lambda 10-3 automatic filter wheels (Sutter Instrument Co., Novato, CA) allowing rapid filter switching. Images were collected using a CoolSNAP HQ2 14-bit CCD camera (Photometrics, Tucson, AZ). All devices were controlled through Metamorph Premier v7.7 software (Molecular Devices, Downingtown, PA). Quantitative analysis of the imaging data and the preparation of presentation quality images were performed using Metamorph v7.7 software. Quantitative stoichiometric FRET analysis of the data was performed with proprietary software created by the CLCI staff using MATLAB R2009a (The Mathworks, Natick, MA), and this FRETcalc software can be obtained from the University of Michigan Tech Transfer. The methods and algorithms used in FRET stoichiometry have been previously described [21,22].

\section{Results and Discussion}

\subsection{Results}

3.1.1. PKCE Phosphorylates and Binds to RhoA. Our laboratory reported that $\mathrm{PKC} \varepsilon$ modulates RhoA activity in HNSCC presumably through posttranslation phosphorylation [8]. In silico prediction of phosphorylation sites identified multiple serine and threonine residues that are putative PKC phosphorylation sites on RhoA suggesting that direct phosphorylation of RhoA through PKCe may be a possibility. To determine if $\mathrm{PKC} \varepsilon$ can directly phosphorylate RhoA, we performed an in vitro kinase reaction and incubated recombinant $\mathrm{PKC} \varepsilon$ with RhoA in the presence of PKC activators, phosphatidylserine and diacylglycerol, and ${ }^{32}$ P-ATP. As shown in Figure 1(a), PKCe directly phosphorylated RhoA. Pro-Q Diamond, a phosphoprotein staining reagent, confirmed RhoA as a substrate for PKC $\varepsilon$ (Figure 1(b)). Next, we identified the phosphorylation sites on RhoA using phosphopeptide mapping 


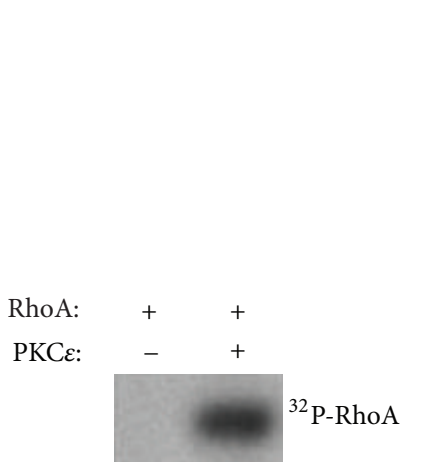

(a)

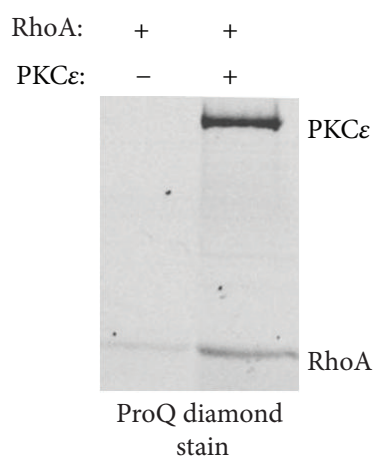

(b)

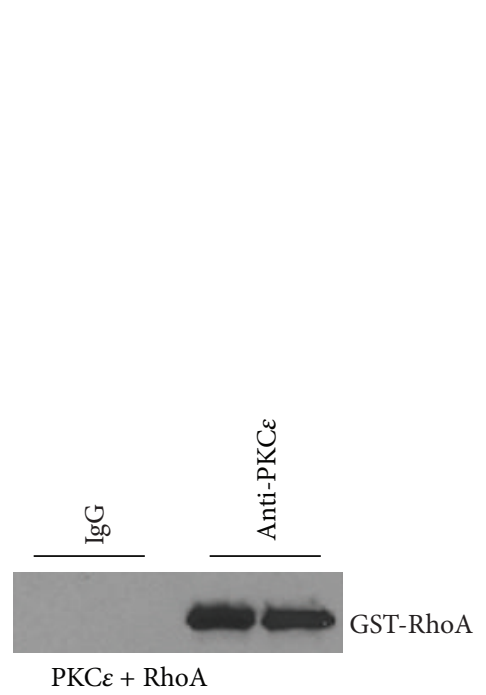

(c)

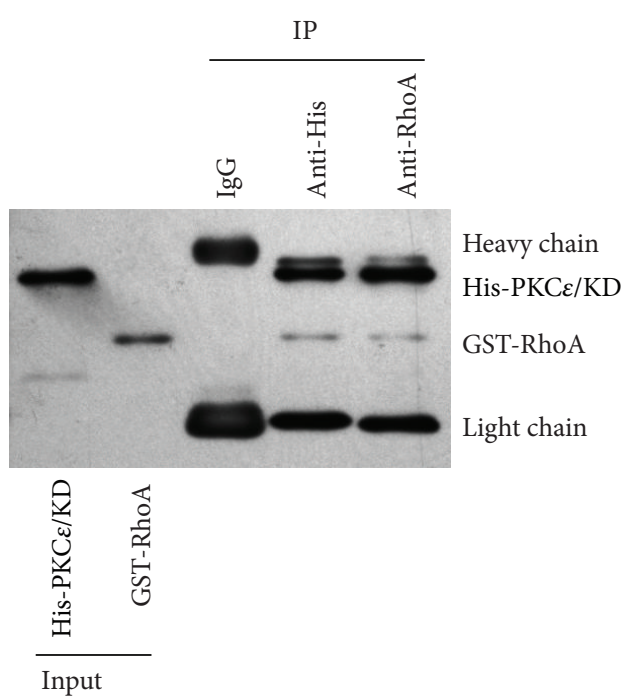

(d)

Figure 1: PKCe phosphorylates and binds to RhoA. (a) PKCe phosphorylates RhoA. Recombinant RhoA was incubated with or without recombinant $\mathrm{PKC} \varepsilon$ in kinase buffer containing PKC activators, phosphatidylserine and diacylglycerol, and $\left[{ }^{32} \mathrm{P}\right] \mathrm{ATP}$ for $30 \mathrm{minutes}$ at $25^{\circ} \mathrm{C}$. Subsequently, the incubation reaction was terminated, separated by SDS-PAGE and visualized using autoradiography. (b) Pro-Q Diamond staining of phosphorylated RhoA. Recombinant RhoA was incubated with or without recombinant PKCe in kinase buffer containing PKC activators, phosphatidylserine and diacylglycerol, and ATP for 30 minutes at $25^{\circ} \mathrm{C}$. Subsequently, the incubation reaction was terminated, separated by SDS-PAGE and visualized using a stain specific to phosphoproteins. (c) PKCe binds to RhoA. Recombinant PKCe was incubated with recombinant RhoA in kinase buffer containing PKC activators, phosphatidylserine and diacylglycerol, for 30 minutes at $25^{\circ} \mathrm{C}$. The binding reaction was immunoprecipitated using agarose-conjugated anti-PKC $\varepsilon$ or nonspecific IgG antibody. The immunoprecipitated proteins were visualized by western blot analysis using an anti-RhoA antibody. Two independent immunoprecipitation experiments are presented. (d) The kinase domain of PKC $\varepsilon$ binds to RhoA. Recombinant His-tagged PKCe-kinase domain was incubated with recombinant GST tagged-RhoA in kinase buffer containing PKC activators, phosphatidylserine and diacylglycerol, for 30 minutes at $25^{\circ} \mathrm{C}$. The binding reaction was immunoprecipitated using agarose-conjugated anti-His, anti-RhoA, or nonspecific IgG antibody. The immunoprecipitated proteins were visualized by western blot analysis using an anti-His and anti-RhoA antibody.

with liquid chromatography-mass spectrometry/mass spectrometry (LC-MS/MS). Phosphorylated RhoA was digested with trypsin, chymotrypsin, or Glu-C. LC-MS ${ }^{\mathrm{E}}$ analysis of peptides resulting from trypsin digested RhoA showed about $83 \%$ coverage, whereas the combined data from trypsin and Glu-C digested RhoA showed 100\% coverage of the serine and threonine residues on RhoA. Thus, it is reasonable to conclude that a comprehensive phosphopeptide map of RhoA was generated using trypsin and Glu-C. Phosphopeptide analysis revealed T127 and S188 as the confident PKCEmediated phosphorylation sites on RhoA. Our results provide the first evidence that RhoA is a direct substrate for PKCE phosphorylation.

3.1.2. PKCE Associates with RhoA. There is limited, although intriguing, literature demonstrating that a kinase preassembles with its substrate prior to a kinase phosphorylation event. The preassembled kinase-substrate complex not only increases specificity but also shortens the time between kinase activation and phosphorylation of the substrate [23, 24]. To determine if $\mathrm{PKC} \varepsilon$ preassembles with RhoA without an active ATP-docked PKCe conformation, recombinant PKC $\varepsilon$ and 


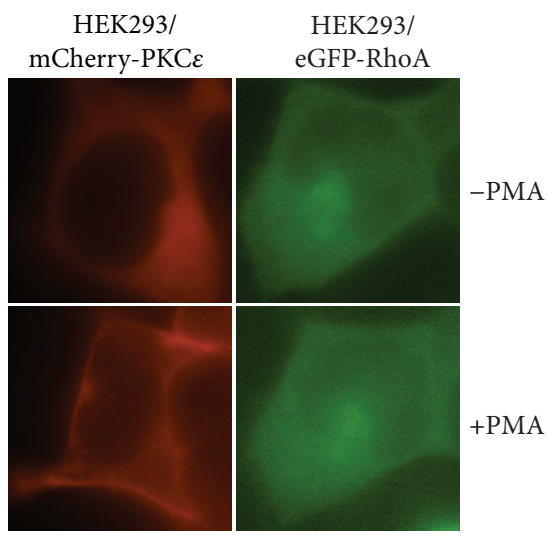

(a)
HEK293/ mCherry-PKCE/eGFP-RhoA

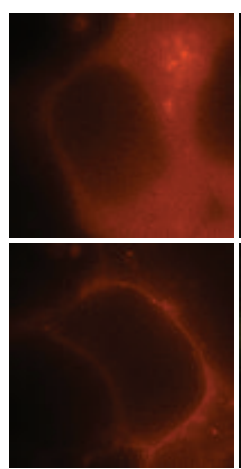

mCherry-PKC $\varepsilon$

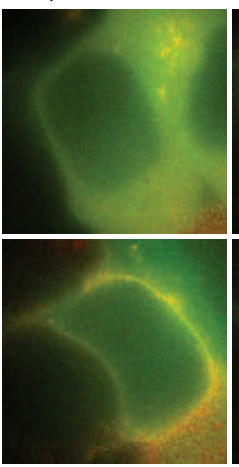

Overlay

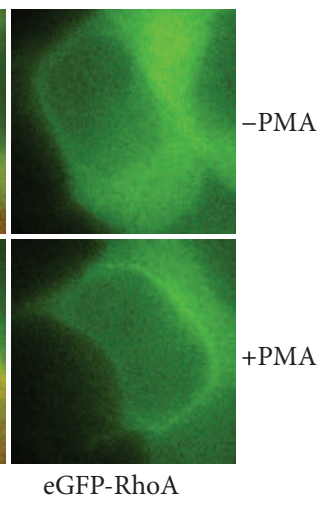

eGFP-RhoA (b)

FIGURE 2: PKCe colocalizes with RhoA at the cell membrane. (a) PKC $\varepsilon$ translocates to the cell membrane, and RhoA remains localized at the cytoplasm in response to PMA. HEK293 cells were transfected with mCherry-PKCe (left panel) or eGFP-RhoA (right panel). Fluorescence images were captured prior to and 15 minutes after PMA $(100 \mathrm{nM})$ stimulation. (b) PKCe colocalizes with RhoA at the cell membrane. HEK293 cells were cotransfected with mCherry-PKC $\varepsilon$ and eGFP-RhoA. Fluorescence images were captured prior to and 15 minutes after PMA (100 nM) stimulation.

RhoA were incubated in ATP-free in vitro kinase buffer. As shown in Figure 1(c), PKCe was able to bind to RhoA under this condition. Moreover, the kinase domain of PKCe was sufficient to bind to RhoA demonstrating that the RhoA docking site is within the PKCe kinase domain (Figure $1(\mathrm{~d})$ ). These results indicate that the binding between $\mathrm{PKC} \varepsilon$ and RhoA does not require an active ATP-docked PKCe kinase conformation, and thus, the interaction between these two proteins is more complex than a transient substrate-kinase intermediate state.

\subsubsection{PKCE Colocalizes with RhoA at the Cell Membrane in} Response to PMA. There is evidence that a preassembled kinase-substrate complex not only enhances phosphorylation specificity and efficiency but also plays a role in cellular localization [25]. To determine if PKC $\varepsilon$ mediates the localization of RhoA, HEK293 cells were transfected with mCherryPKC $\varepsilon$ or eGFP-RhoA or cotransfected with mCherry-PKC $\varepsilon$ and eGFP-RhoA. Subcellular localization of PKC $\varepsilon$ and RhoA was visualized in living cells using fluorescence microscopy in the presence or absence of phorbol 12-myristate 13acetate (PMA). Activation of PKCs with PMA is associated with the translocation of PKCs to the cell membrane. As expected, $\mathrm{PKC} \varepsilon$ was translocated from the cytoplasm to the cell membrane following PMA stimulation in HEK293 cells overexpressing mCherry-PKC $\varepsilon$ (Figure 2(a)). In contrast, in HEK293 cells overexpressing eGFP-RhoA, RhoA remained at the cytoplasm following PMA treatment. As shown in Figure 2(b), PMA treatment induced RhoA to colocalize with PKC $\varepsilon$ at the cell membrane in HEK293 cells transfected to overexpress mCherry-PKC $\varepsilon$ and eGFP-RhoA. Our data showed that $\mathrm{PKC} \varepsilon$ traffics RhoA to the cell membrane following a general PKC activation signal in HEK293 cells.

3.1.4. Dynamic Interaction between PKCE and RhoA in Live Cells. To better define the interaction between PKCE and
RhoA with spatiotemporal resolution, HEK293 cells overexpressing mCherry-PKC $\varepsilon$ and eGFP-RhoA were stimulated with PMA, and images collected over a 12.5 minute time course were subjected to quantitative stoichiometric FRET analysis. PMA treatment induced an obvious reorganization of mCherry-PKCe and eGFP-RhoA in the cell from the cytoplasm to the cell membrane as evidenced by comparing the IA and ID images at $0 \mathrm{~min}$ and $12.5 \mathrm{~min}$ after PMA stimulation, respectively (Figure 3 ). Furthermore, PMAtreatment resulted in an overall increase in ED, a measure of the FRET efficiency of the interaction between mCherry$\mathrm{PKC} \varepsilon$ and eGFP-RhoA. The initial increase and peak in ED occurred in the cytoplasm followed by an elevation of the $\mathrm{PKC} \varepsilon$-RhoA interaction at the cell membrane for the entire time course. Recruitment of RhoA and the increase in FRET activity was especially robust in the actively ruffling regions of the cell (upper and bottom right corners of the cell). Taken together, FRET analysis demonstrated that in response to PMA stimulation, the PKCe-RhoA complex is recruited to the cell membrane over time, and furthermore, the PKCERhoA complex may be preassembled in the cytoplasm prior to translocation to the cell membrane.

3.1.5. Kinase-Inactive PKCE Colocalizes with RhoA at the Cell Membrane. Our in vitro protein binding experiments indicate that an active ATP-docked PKCe confirmation is not required for $\mathrm{PKC} \varepsilon$ to bind to RhoA. The kinase-inactive $\mathrm{PKC} \varepsilon$ mutant (K437R) contains a point mutation in the ATP binding pocket to prevent ATP occupancy. Interestingly, $\mathrm{PKC} \varepsilon / \mathrm{K} 437 \mathrm{R}$ is localized to the cell membrane in unstimulated HEK293 cells overexpressing mCherry-PKCE/K437R (Figure 4(a)). HEK293 cells cotransfected with mCherryPKC $/$ /K437R and eGFP-RhoA showed colocalization of these two proteins at the cell membrane without PMA stimulation. In support of these observations, quantitative stoichiometric 

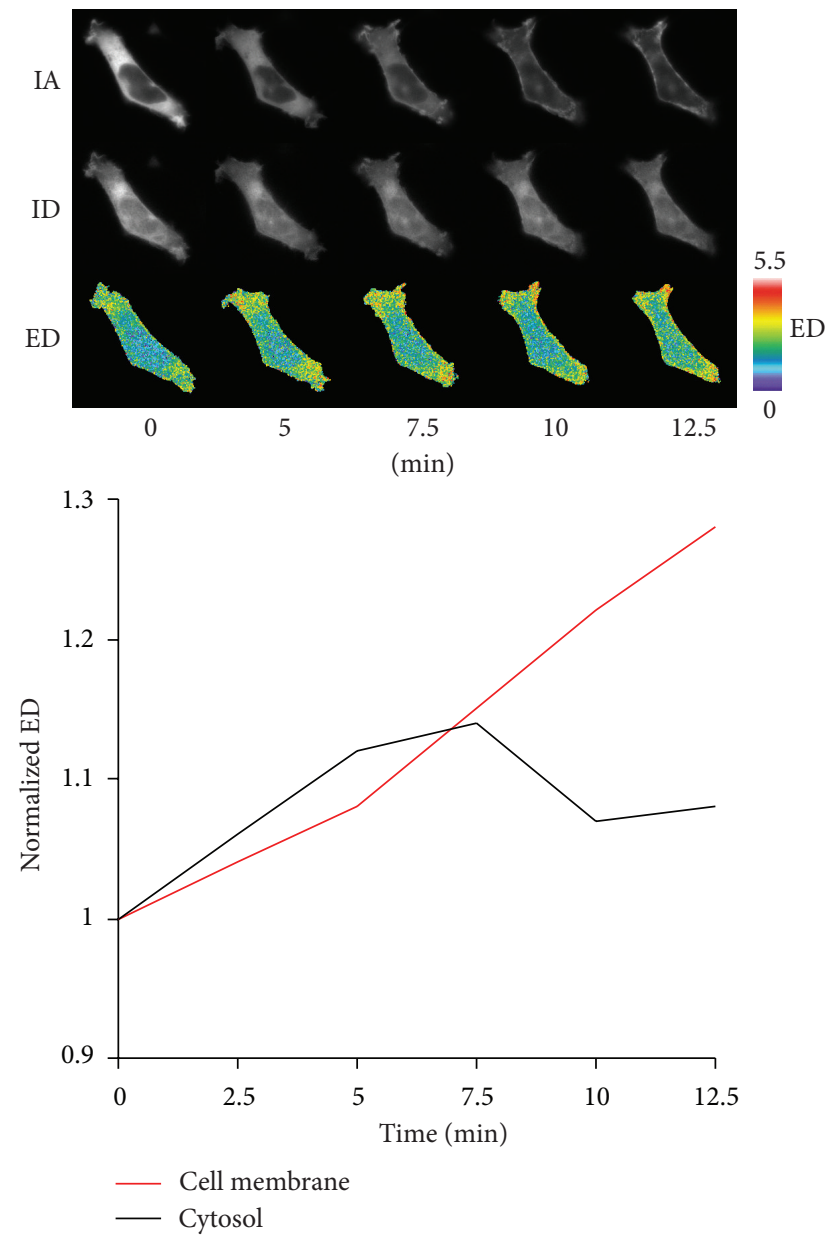

FIGURE 3: Stoichiometric FRET analysis of PKCe-RhoA interaction in live cells. HEK293 cells were cotransfected with mCherry$\mathrm{PKC} \varepsilon$ and eGFP-RhoA. Fluorescence images were captured prior to and every 15 seconds after PMA $(100 \mathrm{nM})$ stimulation for 12.5 minutes and then subjected to quantitative FRET analysis. The images presented at each time point represent mCherry-PKC $\varepsilon$ (acceptor image, IA), eGFP-RhoA (donor image, ID), and the FRET interaction between mCherry-PKCe and eGFP-RhoA (ED). The color bars at the end of the panels indicate the scaling of the ED images with warmer colors representing higher values. Time course of normalized ED for the cell membrane and cytoplasm is presented. ED was normalized to the ED of the cytoplasm at the 0 minute time point. Cell membrane is defined as $\pm 1 \mu \mathrm{m}$ from the cell membrane border. Cytoplasm is defined as all intracellular space, including the nucleus, $1 \mu \mathrm{m}$ from the cell membrane border.

FRET analysis confirmed the interaction between mCherry$\mathrm{PKC} \varepsilon / \mathrm{K} 437 \mathrm{R}$ and eGFP-RhoA at the cell membrane prior to PMA stimulation (Figure 4(b)). The FRET signal at the cell membrane did not change significantly after PMA treatment indicating that the PKCE/K437R-RhoA molecules at that cell compartment were already in complex with each other. These results indicate that the PKCE-RhoA interaction occurs in the absence of ATP and thus do not require an active catalytic site on PKCE. The K437R mutation appears to alter the conformation of $\mathrm{PKC} \varepsilon$ to expose the critical amino acids required to interact with RhoA. Our data confirm that cell membrane localization of RhoA is mediated independently of a PKC $\varepsilon$-RhoA phosphorylation event.

3.2. Discussion. Regulation of RhoA activity is tightly controlled in the GDP/GTP cycle through the coordinated interactions between GTPase activating proteins (RhoGAPs), guanine dissociation inhibitors (RhoGDIs), and guanine nucleotide exchange factors (RhoGEFs). RhoGEFs activate RhoA by catalyzing the exchange of GDP to GTP. RhoGAPs deactivate RhoA by enhancing the intrinsic GTPase activity of RhoA to hydrolyze GTP to GDP. RhoGDIs prevent RhoA activation by sequestering GDP-bound RhoA in the cytoplasm. In addition to this well-described regulatory pathway, there is evidence that posttranslational phosphorylation is an alternate mechanism used to control RhoA. Protein kinase A (PKA) was reported to phosphorylate S188 of RhoA resulting in relocalization of GTP-bound RhoA from the membrane to the cytoplasm, possibly through enhanced interaction with RhoGDI [26]. Protein kinase G (PKG) activation was demonstrated to increase RhoA stability resulting in an increase in total RhoA protein levels [27]. Furthermore, phosphorylation of S188 on RhoA protected RhoA, particularly the GTP-bound active form, from ubiquitin/proteasomemediated degradation [28]. In the present study, PKC $\varepsilon$ was also shown to phosphorylate S188 of RhoA. It is interesting that three different kinases, PKA, PKG, and PKCe, are able to phosphorylate S188 suggesting that phosphorylation of S188 may be a nondiscriminatory mechanism to enhance RhoA stability. In addition to S188, T127 was identified as a novel RhoA phosphorylation site suggesting that PKCe may sequentially phosphorylate RhoA to fine tune RhoA levels and/or activation. Ongoing efforts in our laboratory will delineate the physiological significance of T127 and S188 phosphorylation on RhoA function in HNSCC.

We made the novel observation that recombinant PKCE associates with recombinant RhoA in the absence of ATP indicating that the PKCe-RhoA complex is assembled without an active ATP-docked PKC $\varepsilon$ conformation. Additionally, kinase-inactive $\mathrm{PKC} \varepsilon$ was sufficient to colocalize with RhoA at the cell membrane in live HEK293 cells providing further evidence that the PKCE-RhoA interaction is much more involved than as a transient kinase-substrate transition state. These observations is consistent with a published report demonstrating that the mitogen activated protein kinase (MAPK) substrate complexes are often spatially separate from the kinase active site and the substrate phosphorylation site [23]. The region of the kinase that binds to a substrate has only been identified in two cases, for c-Jun aminoterminal kinase 2 (JNK2) and C-terminal Src kinase (Csk) [29, 30]. The substrate-docking sites for JNK2 and Csk were identified to be within the kinase domain and in proximity, within 50 amino acids, to the catalytic loop of the kinase $[29,30]$. Consistent with these results, the kinase domain of PKCE is sufficient to bind to RhoA. Our work showed that PKCe binds to RhoA within the kinase domain and without the requirement of ATP.

The accepted model of PMA-mediated activation of PKCs is that PMA changes PKCs from a closed to an open 


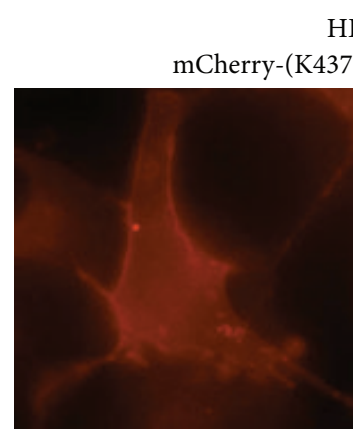

mCherry-(K437R) $\mathrm{PKC} \varepsilon$
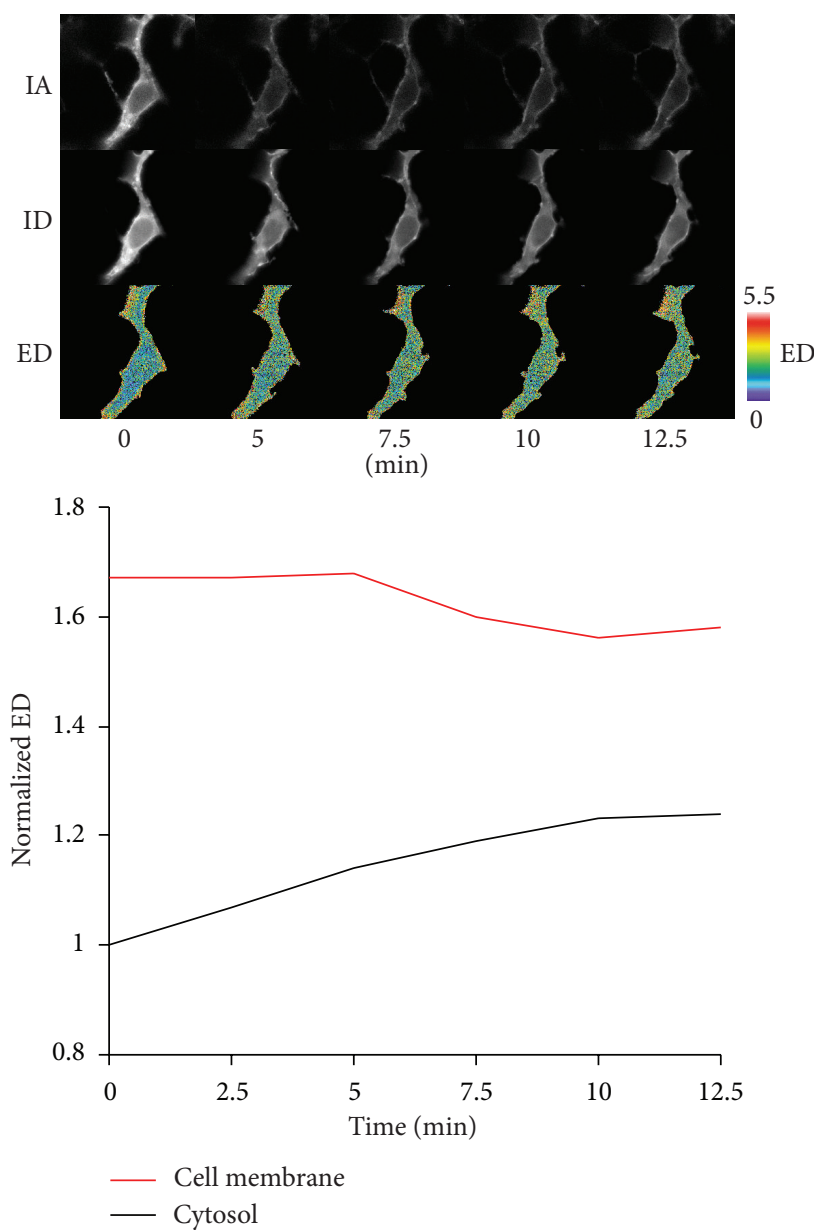

(b)

FIGURE 4: Kinase-inactive PKCe colocalizes with RhoA at the cell membrane. (a) Colocalization of kinase-inactive PKC $\varepsilon$ and RhoA. HEK293 cells were cotransfected with mCherry-PKCE/K437R and eGFP-RhoA. Fluorescence images were captured prior to and 15 minutes after PMA $(100 \mathrm{nM})$ stimulation. (b) Stoichiometric FRET analysis of kinase-inactive PKC $\varepsilon$ and RhoA interaction. HEK293 cells were cotransfected with mCherry-PKC $\varepsilon /$ K437R and eGFP-RhoA. Fluorescence images were captured prior to and every 15 seconds after PMA (100 nM) stimulation for 12.5 minutes and then subjected to quantitative FRET analysis. The images presented at each time point represent mCherry-PKCE/K437R (acceptor image, IA), eGFP-RhoA (donor image, ID), and the FRET interaction between mCherry-PKCe/K437R and eGFP-RhoA (ED). The color bars at the end of the panels indicate the scaling of the ED images with warmer colors representing higher values. Time course of normalized ED for the cell membrane and cytoplasm is presented. ED was normalized to the ED of the cytoplasm at the 0 minute time point. Cell membrane is defined as $\pm 1 \mu \mathrm{m}$ from the cell membrane border. Cytoplasm is defined as all intracellular space, including the nucleus, $1 \mu \mathrm{m}$ from the cell membrane border.

conformation resulting in translocation of PKCs to the cell membrane. FRET results showed that the initial response to PMA is an increase in the molecular interaction between $\mathrm{PKC} \varepsilon$ and RhoA in the cytoplasm. This observation indicates that the active $\mathrm{PKC} \varepsilon$ conformation is required to expose the RhoA docking site and thus allow RhoA to complex with $\mathrm{PKC} \varepsilon$. The interaction between PKC $\varepsilon$ and RhoA showed an early peak at the cytoplasm and then decreased to basal levels for the remainder of the time course. In contrast, a gradual but prolonged increase in FRET intensity was observed at the cell membrane, in particular the actively ruffling regions of the cell, over the entire time course. A plausible explanation is that, following a PKC activation signal, PKCE and RhoA are assembled in the cytoplasm, and the resulting complex is subsequently trafficked to the cell membrane. It is important to point out that PMA does not induce translocation of RhoA to the cell membrane without the presence of PKC $\varepsilon$. Therefore, the recruitment of the PKCE-RhoA complex to the cell membrane is completely dependent on the cellular localization of $\mathrm{PKC} \varepsilon$ in response to a stimulus.

Fluorescence microscopy showed that kinase-inactive $\mathrm{PKC} \varepsilon$ is predominantly localized to the cell membrane under basal conditions. This result suggests that the PKCE/K437R is in an open conformation capable to interact with chaperone proteins involved in PKCE translocation. The cellular localization of RhoA is concentrated at the cell membrane in cells cotransfected with mCherry-PKCe/K437R and eGFPRhoA. Similarly, FRET analysis showed that the interaction 
between $\mathrm{PKC} \varepsilon / \mathrm{K} 437 \mathrm{R}$ and RhoA is concentrated at the cell membrane rather than at the cytoplasm in unstimulated cells. Furthermore, PMA did not alter the extent of the molecular interaction between $\mathrm{PKC} \varepsilon / \mathrm{K} 437 \mathrm{R}$ and RhoA at the cell membrane. FRET analysis with $\mathrm{PKC} \varepsilon / \mathrm{K} 437 \mathrm{R}$ confirmed our in vitro observations and showed that $\mathrm{PKC} \varepsilon$ is able to recruit RhoA to the cell membrane without a PKCERhoA phosphorylation event. Taken together, these findings support a kinase independent role of PKC $\varepsilon$ as a chaperone to traffic RhoA to the cell membrane.

\section{Conclusions}

Work from our laboratory provided the initial evidence linking two proteins, PKCe and RhoA, intimately involved in metastasis. The PKCE-RhoA signaling module was shown to modulate cancer cell invasion and motility. However, the molecular mechanism of $\mathrm{PKC} \varepsilon$ regulation of RhoA remains to be elucidated. In this study, our results revealed that $\mathrm{PKC} \varepsilon$ has both kinase-dependent and kinase-independent functions to regulate RhoA; $\mathrm{PKC} \varepsilon$ directly phosphorylates RhoA and, moreover, serves as a chaperone to translocate RhoA to the cell membrane.

\section{Authors' Contribution}

Tizhi Su designed and conducted most of the experiments and analyzed and interpreted data. Samuel Straight assisted with the FRET experiments and analysis. Liwei Bao and Xiujie Xie assisted with the experiments and analyzed and interpreted data. Caryn L. Lehner and Greg S. Cavey performed the phosphopeptide mapping of RhoA. Theodoros N. Teknos analyzed and interpreted data. Quintin Pan conceived and supervised the project, designed experiments, analyzed and interpreted data, and wrote the paper. All authors read and approved the final paper.

\section{Acknowledgments}

This work was supported in part by the National Cancer Institute at the National Institutes of Health (R01CA135096), American Cancer Society (RSG0821901), and Arthur G. James Cancer Hospital and Richard J. Solove Research Institute, the Ohio State University Comprehensive Cancer Center. The authors acknowledge the Michigan Economic Development Corporation for their financial support of the Southwest Michigan Innovation Center, mass spectrometry lab.

\section{References}

[1] H. Mischak, J. Goodnight, W. Kolch et al., "Overexpression of protein kinase C- $\delta$ and $-\varepsilon$ in NIH 3T3 cells induces opposite effects on growth, morphology, anchorage dependence, and tumorigenicity," Journal of Biological Chemistry, vol. 268, no. 9, pp. 6090-6096, 1993.

[2] G. P. Perletti, M. Folini, H.-C. Lin, H. Mischak, F. Piccinini, and A. H. Tashjian Jr., "Overexpression of protein kinase C epsilon is oncogenic in rat colonic epithelial cells," Oncogene, vol. 12, no. 4, pp. 847-854, 1996.

[3] S. D. Tachado, M. W. Mayhew, G. G. Wescott et al., "Regulation of tumor invasion and metastasis in protein kinase $\mathrm{C}$ epsilontransformed NIH3T3 fibroblasts," Journal of Cellular Biochemistry, vol. 85, no. 4, pp. 785-797, 2002.

[4] A. P. Jansen, E. G. Verwiebe, N. E. Dreckschmidt, D. L. Wheeler, T. D. Oberley, and A. K. Verma, "Protein kinase C- $\varepsilon$ transgenic mice: a unique model for metastatic squamous cell carcinoma," Cancer Research, vol. 61, no. 3, pp. 808-812, 2001.

[5] D. Wu, T. L. Foreman, C. W. Gregory et al., "Protein kinase C $\varepsilon$ has the potential to advance the recurrence of human prostate cancer," Cancer Research, vol. 62, no. 8, pp. 2423-2429, 2002.

[6] F. Benavides, J. Blando, C. J. Perez et al., "Transgenic overexpression of $\mathrm{PKC} \varepsilon$ in the mouse prostate induces preneoplastic lesions," Cell Cycle, vol. 10, no. 2, pp. 268-277, 2011.

[7] Q. Pan, L. W. Bao, C. G. Kleer et al., "Protein kinase Ce is a predictive biomarker of aggressive breast cancer and a validated target for RNA interference anticancer therapy," Cancer Research, vol. 65, no. 18, pp. 8366-8371, 2005.

[8] Q. Pan, L. W. Bao, T. N. Teknos, and S. D. Merajver, “Targeted disruption of protein kinase $\mathrm{C} \varepsilon$ reduces cell invasion and motility through inactivation of RhoA and RhoC GTPases in head and neck squamous cell carcinoma," Cancer Research, vol. 66, no. 19, pp. 9379-9384, 2006.

[9] X. Zhao, L. Lu, N. Pokhriyal et al., "Overexpression of RhoA induces preneoplastic transformation of primary mammary epithelial cells," Cancer Research, vol. 69, no. 2, pp. 483-491, 2009.

[10] L. Ma, Y.-P. Liu, C.-Z. Geng, X.-L. Wang, Y.-J. Wang, and X.-H. Zhang, "Over expression of rhoa is associated with progression in invasive breast duct carcinoma," Breast Journal, vol. 16, no. 1, pp. 105-107, 2010.

[11] S. Valastyan, F. Reinhardt, N. Benaich et al., "A pleiotropically acting microRNA, miR-31, inhibits breast cancer metastasis," Cell, vol. 137, no. 6, pp. 1032-1046, 2009.

[12] D.-D. Dittert, C. Kielisch, I. Alldinger et al., "Prognostic significance of immunohistochemical RhoA expression on survival in pancreatic ductal adenocarcinoma: a high-throughput analysis," Human Pathology, vol. 39, no. 7, pp. 1002-1010, 2008.

[13] D. Wang, K. Dou, H. Xiang et al., "Involvement of RhoA in progression of human hepatocellular carcinoma," Journal of Gastroenterology and Hepatology, vol. 22, no. 11, pp. 1916-1920, 2007.

[14] X. R. Li, F. Ji, J. Ouyang, W. Wu, L. Y. Qian, and K. Y. Yang, "Overexpression of RhoA is associated with poor prognosis in hepatocellular carcinoma," European Journal of Surgical Oncology, vol. 32, no. 10, pp. 1130-1134, 2006.

[15] A. Horiuchi, T. Imai, C. Wang et al., "Up-regulation of small GTPases, RhoA and RhoC, is associated with tumor progression in ovarian carcinoma," Laboratory Investigation, vol. 83, no. 6, pp. 861-870, 2003.

[16] T. Kamai, T. Tsujii, K. Arai et al., "Significant association of Rho/ROCK pathway with invasion and metastasis of bladder cancer," Clinical Cancer Research, vol. 9, no. 7, pp. 2632-2641, 2003.

[17] A. Faried, M. Nakajima, M. Sohda, T. Miyazaki, H. Kato, and H. Kuwano, "Correlation between RhoA overexpression and tumour progression in esophageal squamous cell carcinoma," European Journal of Surgical Oncology, vol. 31, no. 4, pp. 410414, 2005. 
[18] M. T. Abraham, M. A. Kuriakose, P. G. Sacks et al., "Motilityrelated proteins as markers for head and neck squamous cell cancer," Laryngoscope, vol. 111, no. 7, pp. 1285-1289, 2001.

[19] R. Niggeweg, T. Köcher, M. Gentzel et al., "A general precursor ion-like scanning mode on quadrupole-TOF instruments compatible with chromatographic separation," Proteomics, vol. 6, no. 1, pp. 41-53, 2006.

[20] H. Steen, B. Kuster, M. Fernandez, A. Pandey, and M. Mann, "Detection of tyrosine phosphorylated peptides by precursor ion scanning quadrupole TOF mass spectrometry in positive ion mode," Analytical Chemistry, vol. 73, no. 7, pp. 1440-1448, 2001.

[21] A. Hoppe, K. Christensen, and J. A. Swanson, "Fluorescence resonance energy transfer-based stoichiometry in living cells," Biophysical Journal, vol. 83, no. 6, pp. 3652-3664, 2002.

[22] A. D. Hoppe and J. A. Swanson, "Cdc42, Rac1, and Rac2 display distinct patterns of activation during phagocytosis," Molecular Biology of the Cell, vol. 15, no. 8, pp. 3509-3519, 2004.

[23] J. A. Ubersax and J. E. Ferrell Jr., "Mechanisms of specificity in protein phosphorylation," Nature Reviews Molecular Cell Biology, vol. 8, no. 7, pp. 530-541, 2007.

[24] R. M. Biondi and A. R. Nebreda, "Signalling specificity of Ser/Thr protein kinases through docking-site-mediated interactions," Biochemical Journal, vol. 372, part 1, pp. 1-13, 2003.

[25] R. Ben-Levy, S. Hooper, R. Wilson, H. F. Paterson, and C. J. Marshall, "Nuclear export of the stress-activated protein kinase p38 mediated by its substrate MAPKAP kinase-2," Current Biology, vol. 8, no. 19, pp. 1049-1057, 1998.

[26] P. Lang, F. Gesbert, M. Delespine-Carmagnat, R. Stancou, M. Pouchelet, and J. Bertoglio, "Protein kinase A phosphorylation of RhoA mediates the morphological and functional effects of cyclic AMP in cytotoxic lymphocytes," EMBO Journal, vol. 15, no. 3, pp. 510-519, 1996.

[27] V. Sauzeau, M. Rolli-Derkinderen, C. Marionneau, G. Loirand, and P. Pacaud, "RhoA expression is controlled by nitric oxide through cGMP-dependent protein kinase activation," Journal of Biological Chemistry, vol. 278, no. 11, pp. 9472-9480, 2003.

[28] M. Rolli-Derkinderen, V. Sauzeau, L. Boyer et al., "Phosphorylation of serine 188 protects RhoA from ubiquitin/proteasomemediated degradation in vascular smooth muscle cells," Circulation Research, vol. 96, no. 11, pp. 1152-1160, 2005.

[29] T. Kallunki, B. Su, I. Tsigelny et al., "JNK2 contains a specificitydetermining region responsible for efficient c-Jun binding and phosphorylation," Genes and Development, vol. 8, no. 24, pp. 2996-3007, 1994.

[30] S. Lee, X. Lin, N. H. Nam, K. Parang, and G. Sun, "Determination of the substrate-docking site of protein tyrosine kinase C-terminal Src kinase," Proceedings of the National Academy of Sciences of the United States of America, vol. 100, no. 25, pp. 14707-14712, 2003. 


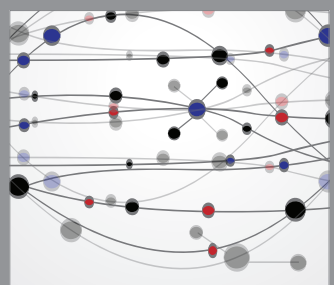

The Scientific World Journal
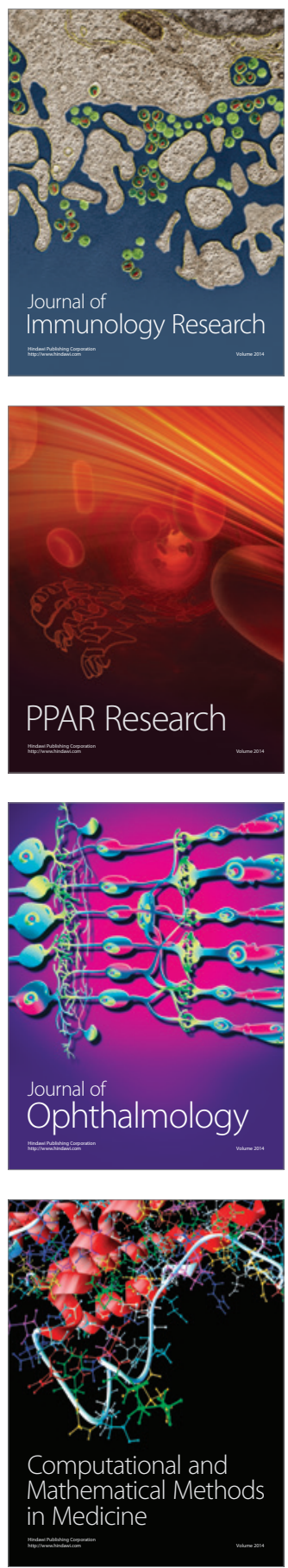

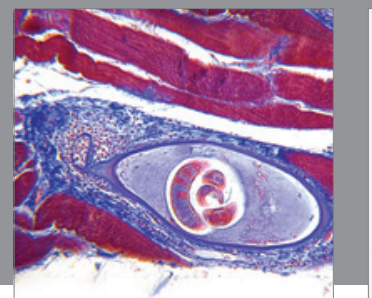

Gastroenterology

Research and Practice
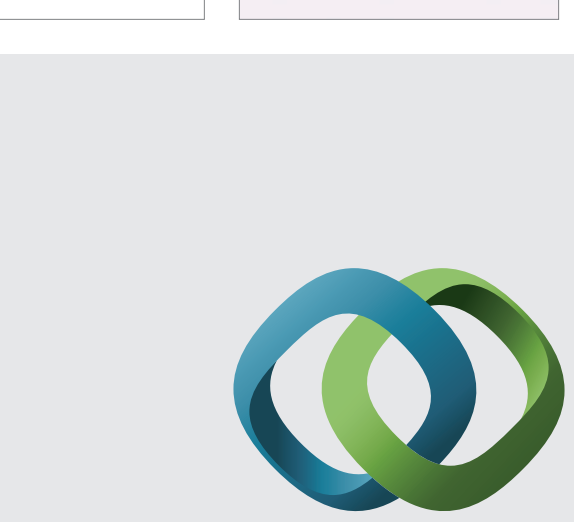

\section{Hindawi}

Submit your manuscripts at

http://www.hindawi.com
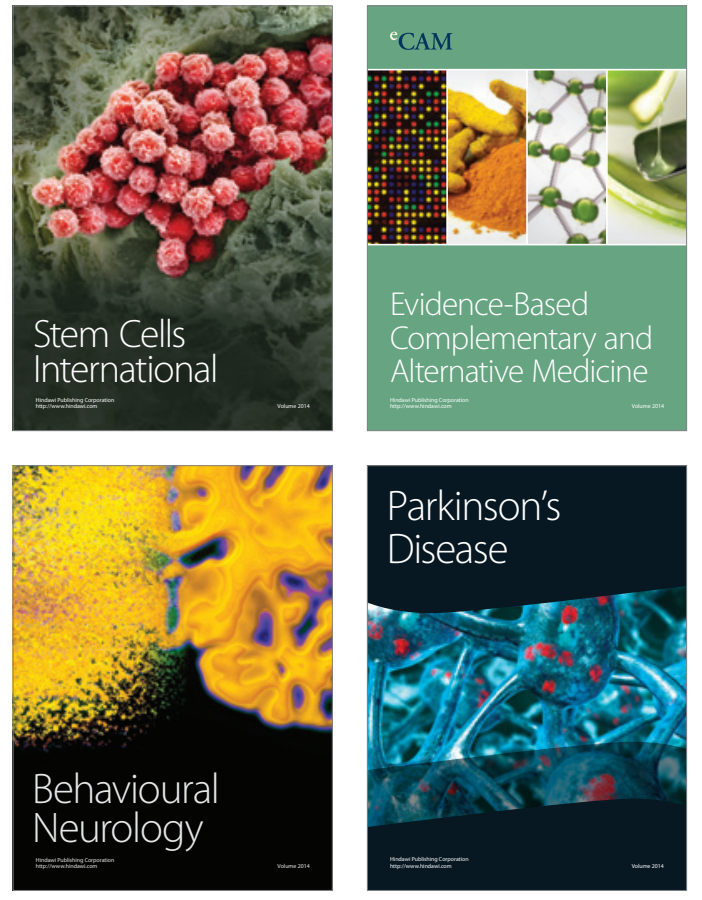
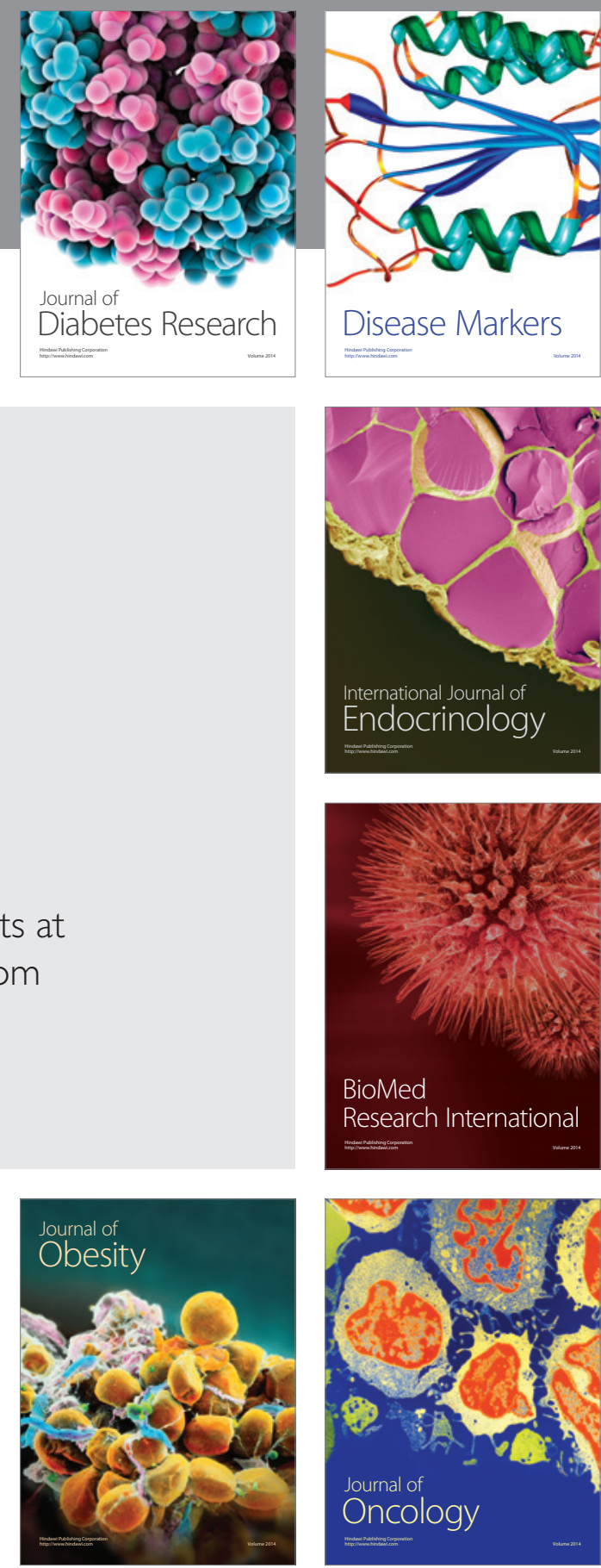

Disease Markers
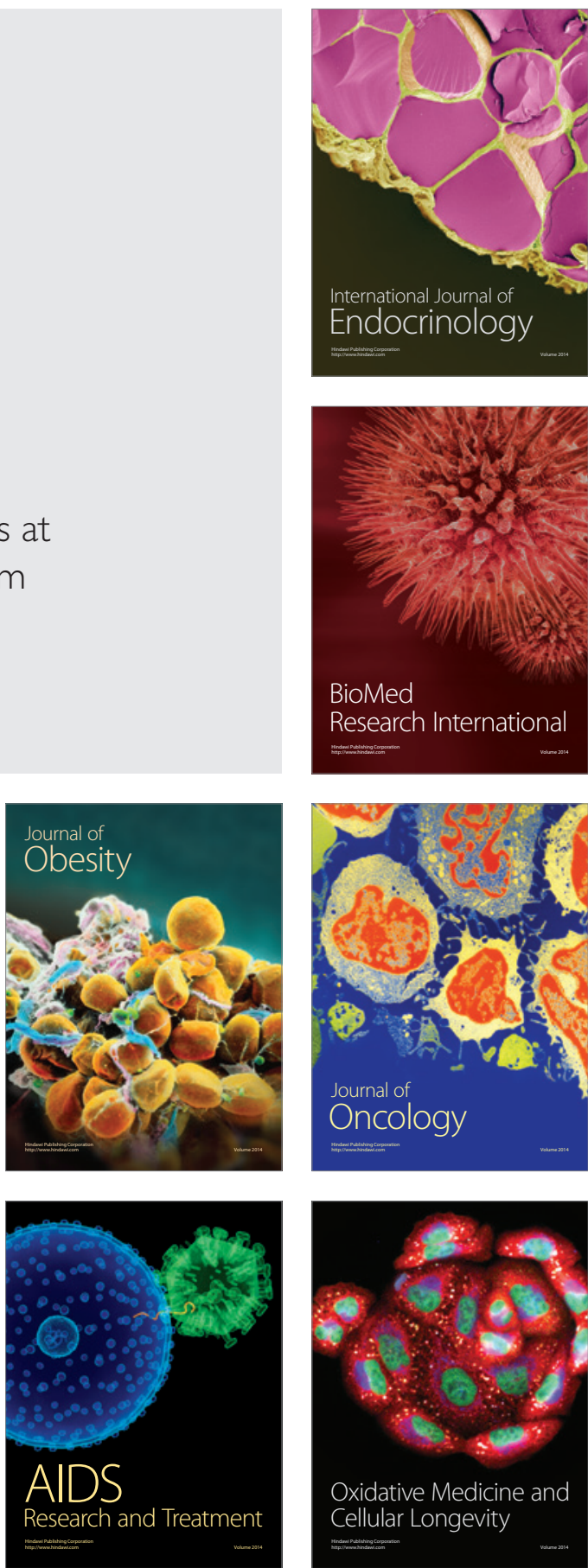